\title{
Risk of Hemorrhage Attributed to Underlying Chronic Diseases and Uninterrupted Aspirin Therapy of Patients Undergoing Minor Oral Surgical Procedures: A Retrospective Cohort Study
}

\author{
Chanapong Rojanaworarit' ${ }^{1}$, Soontaree Limsawan ${ }^{2}$ \\ ${ }^{1}$ Department of Epidemiology, Faculty of Public Health, Mahidol University, Bangkok; ${ }^{2}$ Dental Department, Huayploo Hospital, Nakhon Pathom, \\ Thailand
}

Objectives: This study aimed to estimate the risk of bleeding following minor oral surgical procedures and uninterrupted aspirin therapy in high-risk patients or patients with existing chronic diseases compared to patients who did not use aspirin during minor oral surgery at a public hospital.

Methods: This retrospective cohort study analyzed the data of 2912 patients, aged 20 years or older, who underwent 5251 minor oral surgical procedures at a district hospital in Thailand. The aspirin group was comprised of patients continuing aspirin therapy during oral surgery. The non-aspirin group (reference) included all those who did not use aspirin during surgery. Immediate and late-onset bleeding was evaluated in each procedure. The risk ratio of bleeding was estimated using a multilevel Poisson regression.

Results: The overall cumulative incidence of immediate bleeding was $1.3 \%$ of total procedures. No late-onset bleeding was found. A significantly greater incidence of bleeding was found in the aspirin group (5.8\% of procedures, $p<0.001)$. After adjusting for covariates, a multilevel Poisson regression model estimated that the bleeding risk in the aspirin group was 4.5 times higher than that of the non-aspirin group (95\% confidence interval, 2.0 to 10.0; $p<0.001)$. However, all bleeding events were controlled by simple hemostatic measures.

Conclusions: High-risk patients or patients with existing chronic diseases who continued aspirin therapy following minor oral surgery were at a higher risk of hemorrhage than general patients who had not used aspirin. Nonetheless, bleeding complications were not life-threatening and could be promptly managed by simple hemostatic measures. The procedures could therefore be provided with an awareness of increased bleeding risk, prepared hemostatic measures, and postoperative monitoring, without the need for discontinuing aspirin, which could lead to more serious complications.

Key words: Aspirin, Oral surgery, Hemorrhage, Platelet aggregation inhibitors, Dentistry, Epidemiology

Received: December 25, 2016 Accepted: March 31, 2017

Corresponding author: Chanapong Rojanaworarit, PhD

420/1 Rajwithi, Rajthewi, Bangkok 10400, Thailand

Tel: +66-2-354-8541, Fax: +66-2-354-8562

E-mail: frederick-007@hotmail.com

This is an Open Access article distributed under the terms of the Creative Commons Attribution Non-Commercial License (http://creativecommons.org/licenses/by$\mathrm{nc} / 4.0 /$ ) which permits unrestricted non-commercial use, distribution, and reproduction in any medium, provided the original work is properly cited.

\section{INTRODUCTION}

Current literature suggests that the discontinuation of aspirin therapy prior to minor oral surgery should no longer be practiced, since fatal bleeding rarely occurs and localized bleeding can be controlled by simple hemostatic measures [116]. The paradigm for minor oral surgery of patients with long- 
term aspirin therapy has consequently shifted towards accepting the continuation of aspirin use during surgery and providing similarly practiced procedures to those performed on general patients who do not use aspirin. However, a higher incidence of prolonged bleeding following minor oral surgery in patients with uninterrupted aspirin use compared to those who do not use aspirin during surgery has still been observed in practice-based cohort studies $[8,12]$. Patients prescribed aspirin therapy, in reality, are also more likely to present as highrisk or with existing chronic diseases. This includes being at high risk for cardiovascular events or already having diabetes [17]. Conversely, patients who do not use aspirin have been found to be generally healthier. These findings have raised questions on whether the bleeding risk in high-risk patients or patients with existing chronic diseases who continue aspirin use during surgery can be assumed equivalent to the risk among general patients who receive similarly provided oral surgery but do not use aspirin. Investigation of this issue would provide valuable information on whether the current practice imposes a greater risk of bleeding. If a clinically significant increase of bleeding risk is determined, providing risk information to raise awareness among those performing the surgical procedures will improve surgical care quality in terms of preoperative risk communication to patients, operational preparedness, reductions in tissue damage during surgery, postoperative monitoring for bleeding, and effective management of bleeding occurrences.

Routinely, patients on aspirin therapy undergo a wide variety of oral surgical procedures. Several studies have previously contributed to estimating the bleeding risk specific to dental extractions $[3,8,10-12,16]$, yet only a few have investigated the risk in dental osteotomy [5] or minor oral surgical procedures [7]. Information regarding the overall bleeding risk in patients that undergo various surgical procedures in general dental practice, therefore, remains missing. In addition, while a number of studies have evaluated the bleeding outcome from a single visit for each patient $[5,7,8,10,16]$, individual patient may undergo the same or different surgical procedures over multiple visits. Hence, studies assessing the bleeding occurrence, not only in a single visit, but also from subsequent visits of a specific patient, would provide a more relevant risk estimate.

There is a paucity of information regarding the bleeding risk that is relevant to the current practice in which patients on aspirin therapy undergo various oral surgical procedures in either a single or multiple visits without aspirin interruption.
Therefore, this study was conducted to estimate the risk of prolonged bleeding following minor oral surgery in high-risk patients or patients with existing chronic diseases who continued aspirin therapy during surgery as compared to patients who did not use aspirin during surgery.

\section{METHODS}

\section{Study Setting and Patients}

This study was carried out at Huayploo Hospital, a district health facility in Nakhon Chai Si, Nakhon Pathom, Thailand. This public hospital is a 60 -bed inpatient facility that provides primary and secondary dental care, primarily for Thai residents and additionally for foreign immigrants in nearby communities. Since this study aimed to generalize its results primarily to Thai residents who lived in eligible areas serviced by this health facility, all immigrants were excluded. All Thai dental patients aged 20 years or older who underwent minor oral surgery in a single or multiple visits from January 1, 2013 to December 31, 2015 were initially included. Verification of living in the hospital's serviced areas was further undertaken through review of the hospital registry in which each patient's address was available based on the official civil registration system. Data of 2912 Thai adult patients (5251 procedures) were ultimately retrieved for analysis.

\section{Data Collection Approach}

Data were collected retrospectively. Clinical characteristics, procedural details of the minor oral surgery, and immediate and late-onset bleeding outcomes were retrospectively identified from hospital records for each visit of all individual patients. In this setting, patients at risk of developing a thromboembolic event (e.g., patients with diabetes mellitus or cardiovascular disease) were prescribed single antiplatelet therapy using 80 to $300 \mathrm{mg}$ of aspirin daily. Dual antiplatelet therapy using clopidogrel (Plavix ${ }^{\top \mathrm{M}}$ ) together with aspirin was seldom indicated in this facility, and none of the included patients were found to receive this regimen. Patients were divided into two groups - aspirin and non-aspirin - according to their status of aspirin use. The aspirin use was initially verified through the physician's prescription records and revalidated through routine medical history notes, indicating uninterrupted use prior to dental treatment in each visit. Patients with continued aspirin use were grouped into the aspirin group and their counterparts were collectively regarded as the non-aspirin 
group (reference). Patients taking aspirin commonly presented as high-risk or with existing chronic diseases, while those who did not take aspirin were generally healthier. Therefore, this study did not intend to compare the bleeding risk of aspirin discontinuation against uninterrupted use prior to oral surgery. Specification of patients to the aspirin and non-aspirin groups was relevant to this setting, as discontinuation of aspirin use before oral surgery was no longer practiced and patients with the uninterrupted aspirin therapy underwent minor oral surgery in the same way as their counterparts.

All minor oral surgical procedures routinely provided in this setting were analyzed. These procedures varied in the level of invasiveness. Mildly invasive treatments included plaque and calculus removal by full-mouth scaling and localized alveoloplasty using rongeur forceps or bone files. Moderately invasive procedures comprised root planing and simple or complicated extractions involving single or multiple teeth, with or without periosteal flap elevation. Intensely invasive interventions consisted of the surgical removal of impacted teeth with periosteal flap elevation. For ease of analysis, a single or multiple surgical treatments during one visit were collectively regarded as one procedure. Administration of 2\% lidocaine with 1:100 000 epinephrine was typical for all cases that underwent root planing, dental extraction, and surgical removal of impacted teeth, ensuring the hemostatic effect of epinephrine.

The outcome in this study included immediate and late-onset bleeding. Immediate bleeding was indicated by a record of excessive blood that could not be controlled by gauze packing within 30 minutes postoperatively $[8,10,18]$. A record of returning to the hospital for unstopped bleeding was used to indicate late-onset bleeding [9].

\section{Statistical Analysis}

Patient characteristics and procedural details of oral surgery were summarized using descriptive statistics. An independent samples $t$-test and a two-sample Wilcoxon rank-sum test were used to estimate the differences in a variety of continuous variables between the two samples. The assumption of homogeneity of variance was assessed prior to the application of the $t$-test. An exact probability test was applied to analyze categorical variables. A stratified analysis comparing the cumulative incidences of bleeding between the aspirin and non-aspirin groups by categories of prognostic variables was undertaken. A risk ratio was included as a measure of effect of underlying systemic conditions and uninterrupted aspirin use on bleeding. Since patients visited the facility either once or multiple times within the study period to receive oral surgery provided by the same set of clinicians, bleeding occurrence observed in patients might correlate with each corresponding clinician and independence of these observations could not be assumed [19]. A multilevel Poisson regression [20] was thus applied to statistically explore the interaction effect of aspirin use with each of the other prognostic factors on the occurrence of bleeding in addition to the aforementioned stratified analysis. It was also used to estimate the crude and adjusted risk ratios by taking the correlated nature of data into account [19] and specifying each clinician as a source of correlation. For the multivariable model, an explanatory modeling strategy was adopted to estimate the risk ratio after adjusting for sex, age, diabetes, hypertension, cardiovascular disease, stroke, asthma or chronic obstructive pulmonary disease (COPD), dental professional, surgical procedure, number of extracted teeth, mandible involvement, position of involved teeth, and visit [21].

\section{Ethical Considerations}

This study protocol was considered and approved by the Ethical Review Committee for Human Research, Faculty of Public Health, Mahidol University.

\section{RESULTS}

Patients with continued aspirin use accounted for $11.5 \%$ of all patients undergoing oral surgery during the study period. Clinical characteristics were analyzed for all patients at their first visit (baseline) as well as for all visits combined in order to consider the potential changes in clinical status during subsequent visits. Patients in the aspirin group were significantly older and had considerably greater proportions of systemic diseases, with the exception of asthma or COPD (Table 1).

The proportions of oral surgical procedures provided by dental hygienists and dentists were not significantly different between the two groups. Simple extraction was the most common procedure in the aspirin group (49.1\%) while fullmouth scaling was the most common in the non-aspirin group (46.0\%). Roughly $66.5 \%$ of procedures in the aspirin group were related to tooth removal, while only $45.4 \%$ of the same procedures were found in the non-aspirin group. None of the patients in the aspirin group underwent surgical removal of impacted teeth. For both the aspirin and non-aspirin 
Table 1. Clinical characteristics of patients pursuing minor oral surgical procedures

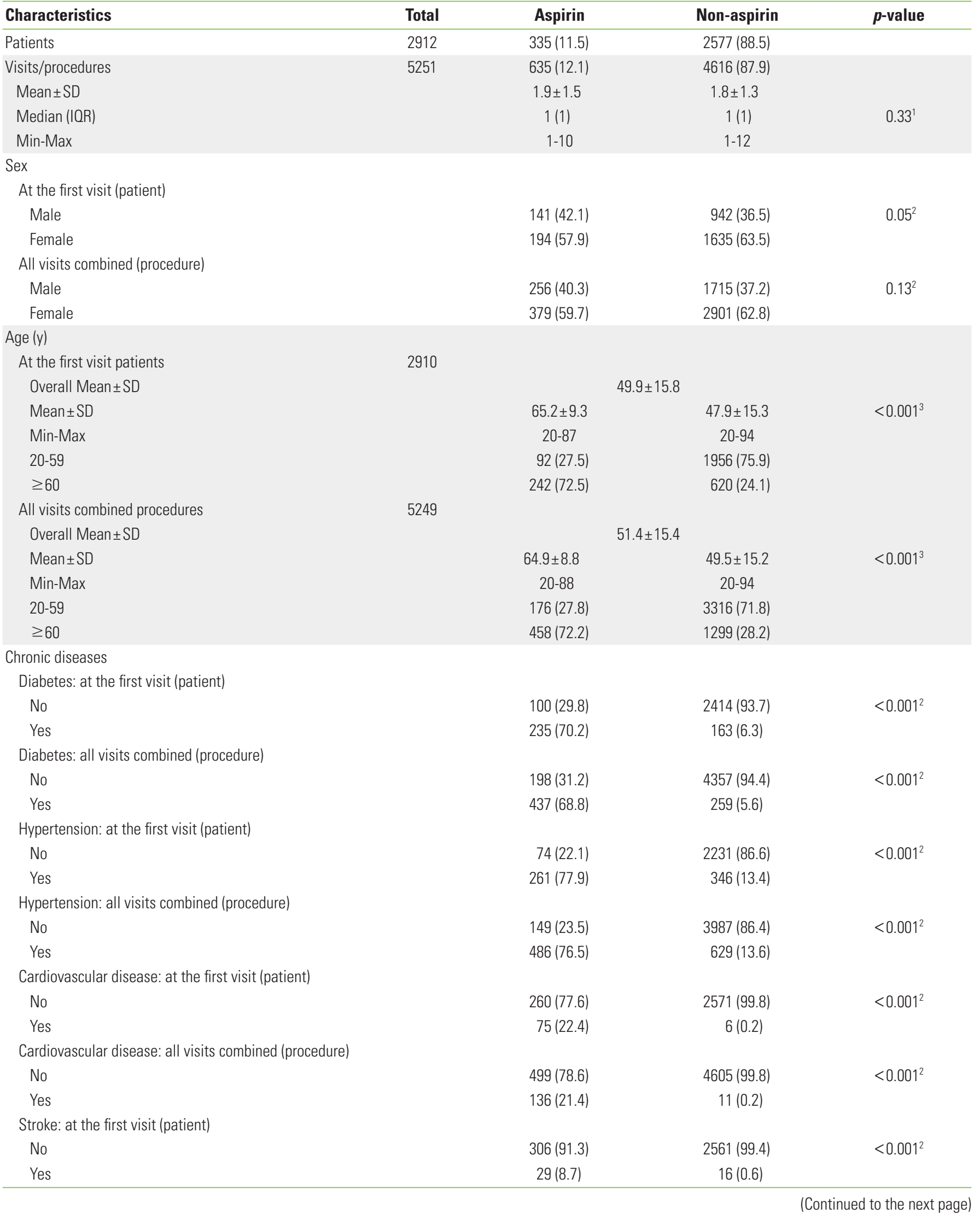


Table 1. Continued from the previous page

\begin{tabular}{|c|c|c|c|c|}
\hline Characteristics & Total & Aspirin & Non-aspirin & $p$-value \\
\hline \multicolumn{5}{|c|}{ Stroke: all visits combined (procedure) } \\
\hline No & & $574(90.4)$ & 4591 (99.5) & $<0.001^{2}$ \\
\hline Yes & & $61(9.6)$ & $25(0.5)$ & \\
\hline \multicolumn{5}{|c|}{ Asthma/COPD: at the first visit (patient) } \\
\hline No & & $329(98.2)$ & $2540(98.6)$ & $0.63^{2}$ \\
\hline Yes & & $6(1.8)$ & $37(1.4)$ & \\
\hline \multicolumn{5}{|c|}{ Asthma/COPD: all visits combined (procedure) } \\
\hline No & & $625(98.4)$ & $4543(98.4)$ & $1.00^{2}$ \\
\hline Yes & & $10(1.6)$ & $73(1.6)$ & \\
\hline \multicolumn{5}{|c|}{ Free of diseases above: at the first visit (patient) } \\
\hline No & & $323(96.4)$ & $451(17.5)$ & $<0.001^{2}$ \\
\hline Yes & & $12(3.6)$ & $2126(82.5)$ & \\
\hline \multicolumn{5}{|c|}{ Free of diseases above: all visits combined (procedure) } \\
\hline No & & $614(96.7)$ & $806(17.5)$ & $<0.001^{2}$ \\
\hline Yes & & $21(3.3)$ & 3810 (82.5) & \\
\hline
\end{tabular}

Values are presented as number (\%).

COPD, chronic obstructive pulmonary disease; IQR, interquartile range; Min, minimum; Max, maximum.

${ }^{1}$ Two-sample Wilcoxon rank-sum test.

${ }^{2}$ Exact probability test.

${ }^{3}$ Independent samples $t$-test.

group, the number of removed teeth ranged from 1 to 6 teeth per visit. The average number of removed teeth per visit in the aspirin group was found to be higher than that in the non-aspirin group $(p<0.001)$. For both groups, the main position of removed teeth was in the molar segment. Surgical procedures in the aspirin group mostly involved the mandible only (39.6\%), while the most common procedure in the non-aspirin group was found to involve the full mouth, mostly by scaling (39.3\%) (Table 2).

The overall cumulative incidence of immediate bleeding was $1.3 \%$ of total procedures. No late-onset bleeding was found. A significantly greater incidence of immediate bleeding was found in the aspirin group ( $5.8 \%$ of procedures) compared to that of the non-aspirin group $(0.7 \%, p<0.001)$. A stratified analysis of bleeding incidence by prognostic factors revealed that bleeding incidences in the aspirin group were considerably greater than in the non-aspirin group, specifically among males, elderly patients, diabetic patients, and those who had undergone a simple extraction. Bleeding in the non-aspirin group occurred neither among those with cardiovascular disease, stroke, or asthma/COPD; nor in those receiving root planing or alveoloplasty. From the overall total of 70 bleeding events, 45 (64.3\%) were observed among cases that received simple extractions, while the rest occurred during other proce- dures, including scaling, complicated extraction, root planing, surgical removal of impacted teeth, and alveoloplasty. No bleeding occurred during extractions of more than three teeth, in procedures involving the full mouth, or in those involving some quadrants in both arches. All bleeding events were effectively controlled by local hemostatic measures thereafter.

Interaction effects of aspirin use with other prognostic factors on the occurrence of bleeding could be statistically assessed only when the bleeding outcome occurred in all strata of the stratified prognostic factor. Some prognostic factors, such as cardiovascular disease, stroke, or the full mouth involved in minor surgery, had no bleeding outcome in one of their stratified strata and thus could not be statistically assessed for the interaction effect. Only the interaction between aspirin use and surgery involving the mandible only was found to be statistically significant $(p<0.05)$ (Table 3 ).

The crude risk ratio of each prognostic factor is presented in Table 4. From the univariable analysis, occurrence of bleeding in the aspirin group was 7.7 times more likely than that of the non-aspirin group $(p<0.001)$. Age, diabetes mellitus, hypertension, cardiovascular disease, surgical procedure, number of extracted teeth, and procedures involving the mandible only were also found to significantly increase bleeding risk. However, no association was determined between procedures involv- 
Table 2. Service providers and procedural details of minor oral surgery

\begin{tabular}{|c|c|c|c|}
\hline Characteristics & Aspirin & Non-aspirin & $p$-value \\
\hline Procedures $(n=5251)$ & $635(12.1)$ & 4616 (87.9) & \\
\hline \multicolumn{4}{|l|}{ Dental professional } \\
\hline Dental hygienist & $272(42.8)$ & $1835(39.7)$ & $0.14^{1}$ \\
\hline Dentist & $363(57.2)$ & $2781(60.3)$ & \\
\hline \multicolumn{4}{|l|}{ Oral surgical procedure } \\
\hline 1. Scaling & $161(25.3)$ & $2125(46.0)$ & $<0.001^{2}$ \\
\hline 2. Simple extraction & $312(49.1)$ & $1355(29.3)$ & \\
\hline 3. Complicated extraction & $79(12.4)$ & $549(11.8)$ & \\
\hline 4. Root planing & $44(6.9)$ & $376(8.2)$ & \\
\hline 5. Impacted tooth surgery & $0(0.0)$ & $104(2.3)$ & \\
\hline 6. Alveoloplasty & $8(1.3)$ & $17(0.4)$ & \\
\hline \multicolumn{4}{|l|}{ 7. Multiple procedures: } \\
\hline $1 \& 2$ & $18(2.8)$ & $32(0.7)$ & \\
\hline $1 \& 3$ & $1(0.2)$ & $5(0.1)$ & \\
\hline $2 \& 3$ & $5(0.8)$ & $22(0.5)$ & \\
\hline $2 \& 4$ & $5(0.8)$ & $3(0.1)$ & \\
\hline $2 \& 5$ & $0(0.0)$ & $14(0.3)$ & \\
\hline $3 \& 5$ & $0(0.0)$ & $14(0.3)$ & \\
\hline $3 \& 6$ & $1(0.2)$ & $0(0.0)$ & \\
\hline $5 \& 6$ & $1(0.2)$ & $0(0.0)$ & \\
\hline \multicolumn{4}{|l|}{ Extent of surgical involvement } \\
\hline \multicolumn{4}{|l|}{ Tooth removal } \\
\hline No & $213(33.5)$ & $2519(54.6)$ & $<0.001^{1}$ \\
\hline Yes ( $\geq 1$ tooth) & 422 (66.5) & $2097(45.4)$ & \\
\hline \multicolumn{4}{|l|}{ No. of removed teeth per visit } \\
\hline Mean \pm SD & $1.4 \pm 0.8$ & $1.2 \pm 0.6$ & $<0.001^{3}$ \\
\hline 1 & $299(70.9)$ & $1760(83.9)$ & \\
\hline 2 & $85(20.1)$ & $273(13.0)$ & \\
\hline 3 & $29(6.9)$ & $41(2.0)$ & \\
\hline 4 & $5(1.2)$ & $14(0.7)$ & \\
\hline 5 & $3(0.7)$ & $6(0.3)$ & \\
\hline 6 & $1(0.2)$ & $3(0.1)$ & \\
\hline \multicolumn{4}{|l|}{ Position of removed teeth in dental arch } \\
\hline Anterior segment & $143(33.9)$ & $369(17.6)$ & $<0.001^{1}$ \\
\hline Premolar segment & $106(25.1)$ & $426(20.3)$ & \\
\hline Molar segment & $172(40.8)$ & $1296(61.8)$ & \\
\hline Anterior and premolar & $1(0.2)$ & $0(0.0)$ & \\
\hline Anterior and molar & $0(0.0)$ & $1(0.1)$ & \\
\hline Premolar and molar & $0(0.0)$ & $5(0.2)$ & \\
\hline \multicolumn{4}{|l|}{ Involved dental arch } \\
\hline Maxilla only (1st or 2nd quadrant or both) & $234(36.9)$ & $1351(29.3)$ & $<0.001^{1}$ \\
\hline Mandible only (3rd or 4th quadrant or both) & $252(39.6)$ & $1326(28.7)$ & \\
\hline Both arches (some quadrants involved) & $9(1.4)$ & $126(2.7)$ & \\
\hline Full mouth (all quadrants involved) & $140(22.1)$ & $1813(39.3)$ & \\
\hline
\end{tabular}

Values are presented as number (\%).

${ }^{1}$ Exact probability test.

${ }^{2}$ Chi-squared test.

${ }^{3}$ Independent samples $t$-test (unequal variances). 
Table 3. Cumulative incidence of bleeding following oral surgical procedures

\begin{tabular}{|c|c|c|c|c|c|}
\hline Characteristics & Total & Aspirin & Non-aspirin & $p$-value ${ }^{1}$ & $\begin{array}{l}p \text {-value for } \\
\text { interaction }^{2}\end{array}$ \\
\hline Procedures & 5251 & $635(12.1)$ & 4616 (87.9) & & \\
\hline \multicolumn{6}{|l|}{ Bleeding (overall incidence: $1.3 \%$ of all procedures) } \\
\hline No & & $598(94.2)$ & $4583(99.3)$ & $<0.001$ & \\
\hline Yes & & $37(5.8)$ & $33(0.7)$ & & \\
\hline \multicolumn{6}{|c|}{$\begin{array}{l}\text { Stratified analysis of bleeding incidence by prognostic factor } \\
\text { ( } \mathrm{n}=\text { procedures) }\end{array}$} \\
\hline Sex & & & & & 0.41 \\
\hline Male & $1971(37.5)$ & $16(6.3)$ & $16(0.9)$ & $<0.001$ & \\
\hline Female & $3280(62.5)$ & $21(5.5)$ & $17(0.6)$ & $<0.001$ & \\
\hline Age (y) & & & & & 0.59 \\
\hline $20-59$ & $3492(66.5)$ & $9(5.1)$ & $21(0.6)$ & $<0.001$ & \\
\hline$\geq 60$ & $1757(33.5)$ & $28(6.1)$ & $12(0.9)$ & $<0.001$ & \\
\hline Diabetes & & & & & 0.15 \\
\hline No & $4555(86.7)$ & $16(8.1)$ & $30(0.7)$ & $<0.001$ & \\
\hline Yes & $696(13.3)$ & $21(4.8)$ & $3(1.2)$ & 0.01 & \\
\hline Hypertension & & & & & 0.47 \\
\hline No & 4136 (78.8) & $7(4.7)$ & $25(0.6)$ & $<0.001$ & \\
\hline Yes & $1115(21.2)$ & $30(6.2)$ & $8(1.3)$ & $<0.001$ & \\
\hline \multicolumn{6}{|l|}{ Cardiovascular disease } \\
\hline No & $5104(97.2)$ & $24(4.8)$ & $33(0.7)$ & $<0.001$ & \\
\hline Yes & $147(2.8)$ & $13(9.6)$ & $0(0.0)$ & 0.60 & \\
\hline \multicolumn{6}{|l|}{ Stroke } \\
\hline No & $5165(98.4)$ & $34(5.9)$ & $33(0.7)$ & $<0.001$ & \\
\hline Yes & $86(1.6)$ & $3(4.9)$ & $0(0.0)$ & 0.55 & \\
\hline \multicolumn{6}{|l|}{ Asthma/COPD } \\
\hline No & 5168 (98.4) & $36(5.8)$ & $33(0.7)$ & $<0.001$ & \\
\hline Yes & $83(1.6)$ & $1(10.0)$ & $0(0.0)$ & 0.12 & \\
\hline Dental professional & & & & & 0.30 \\
\hline Dental hygienist & $2107(40.1)$ & $14(5.2)$ & $16(0.9)$ & $<0.001$ & \\
\hline Dentist & $3144(59.9)$ & $23(6.3)$ & $17(0.6)$ & $<0.001$ & \\
\hline Surgical procedure & & & & & 0.23 \\
\hline Simple extraction [+/- scaling or root planing] & $1744(33.2)$ & $25(7.5)$ & $20(1.4)$ & $<0.001$ & \\
\hline Simple extraction only & 1667 & $24(7.7)$ & $18(1.3)$ & $<0.001$ & \\
\hline Simple extraction and root planing & 27 & $1(20.0)$ & $2(9.1)$ & 0.47 & \\
\hline All other procedures & $3507(66.8)$ & $12(4.0)$ & $13(0.4)$ & $<0.001$ & \\
\hline Scaling & 2286 & $1(0.6)$ & $1(0.1)$ & 0.14 & \\
\hline Complicated extraction & 628 & $9(11.3)$ & $11(2.0)$ & $<0.001$ & \\
\hline Root planing & 420 & $1(2.3)$ & $0(0.0)$ & 0.10 & \\
\hline Impacted tooth & 104 & $0(0.0)$ & $1(1.0)$ & & \\
\hline Alveoloplasty & 25 & $1(12.5)$ & $0(0.0)$ & 0.32 & \\
\hline \multicolumn{6}{|l|}{ No. of extracted teeth } \\
\hline None & 2732 (52.0) & $3(1.4)$ & $1(0.04)$ & 0.002 & \\
\hline 1 & 2059 (39.2) & $33(11.0)$ & $31(1.8)$ & $<0.001$ & \\
\hline 2 & $358(6.9)$ & $1(1.2)$ & $1(0.3)$ & 0.42 & \\
\hline$\geq 3$ & $102(1.9)$ & $0(0.0)$ & $0(0.0)$ & & \\
\hline
\end{tabular}


Table 3. Continued from the previous page

\begin{tabular}{|c|c|c|c|c|c|}
\hline Characteristics & Total & Aspirin & Non-aspirin & $p$-value ${ }^{1}$ & $\begin{array}{l}p \text {-value for } \\
\text { interaction }\end{array}$ \\
\hline \multicolumn{6}{|l|}{ Full mouth involved } \\
\hline No & $3298(62.8)$ & $37(7.5)$ & $33(1.2)$ & $<0.001$ & \\
\hline Yes & 1953 (37.2) & $0(0.0)$ & $0(0.0)$ & & \\
\hline Only maxilla involved & & & & & 0.56 \\
\hline No & $3664(69.8)$ & $23(5.7)$ & $25(0.8)$ & $<0.001$ & \\
\hline Yes & $1585(30.2)$ & $14(6.0)$ & $8(0.6)$ & $<0.001$ & \\
\hline Only mandible involved & & & & & 0.03 \\
\hline No & $3671(70.0)$ & $14(3.7)$ & $8(0.2)$ & $<0.001$ & \\
\hline Yes & $1578(30.0)$ & $23(9.1)$ & $25(1.9)$ & $<0.001$ & \\
\hline \multicolumn{6}{|l|}{ Some quadrants in both arches } \\
\hline No & $5116(97.4)$ & $37(5.9)$ & $33(0.7)$ & $<0.001$ & \\
\hline Yes & $135(2.6)$ & $0(0.0)$ & $0(0.0)$ & & \\
\hline Position of involved teeth & & & & & 0.33 \\
\hline Anterior segment only & $512(20.3)$ & $9(6.3)$ & $6(1.6)$ & 0.008 & \\
\hline Premolar [+/- anterior] segment & $533(21.2)$ & $5(4.7)$ & $4(0.9)$ & 0.02 & \\
\hline Molar [+/- anterior or premolar] segment & $1474(58.5)$ & $20(11.6)$ & $22(1.7)$ & $<0.001$ & \\
\hline Visit & & & & & 0.95 \\
\hline 1 (first) visit only & $2912(55.5)$ & $22(6.6)$ & $21(0.8)$ & $<0.001$ & \\
\hline$>1$ visit within period & $2339(44.5)$ & $15(5.0)$ & $12(0.6)$ & $<0.001$ & \\
\hline
\end{tabular}

Values are presented as number or number (\%).

${ }^{1}$ Exact probability test.

${ }^{2}$ Interaction of aspirin use and each of other factors estimated by multilevel Poisson regression.

ing the maxilla only and bleeding (risk ratio, $1.0 ; p=0.90$ ), (Table 4). After adjusting for covariates, the multilevel Poisson regression model estimated that the bleeding risk in the aspirin group was 4.5 times more likely than that of the non-aspirin group $(p<0.001)$ (Table 4$)$.

\section{DISCUSSION}

Aspirin use has increasingly become more common among dental patients, particularly those of advanced age. It is primarily used as an antiplatelet prophylaxis to prevent cardiovascular disease [22] but also for the prevention of atherothrombotic vascular complications [3,10,23], such as myocardial infarction. The antiplatelet effect of aspirin can be observed between doses of 40 and $320 \mathrm{mg} / \mathrm{d}$ [10]. In this study, patients in the aspirin group continued to take 80 to $300 \mathrm{mg}$ of aspirin daily during oral surgery, maintaining the antiplatelet effect. The lifesaving benefit of aspirin in secondary prevention of occlusive vascular events definitely outweighs bleeding risk [24]. However, it has been suggested that aspirin use for primary prevention of cardiovascular disease should still be weighed against an increased risk of bleeding [3,24]. As current literature and practice favor the continuation of aspirin therapy during oral surgery [1-16], the risk of thromboembolism may be minimized with the antiplatelet effects of aspirin, while bleeding risk would presumably increase. However, controversy continues to exist on whether bleeding could be more likely to occur. In addition, patients prescribed aspirin therapy in this study were found to present with more systemic diseases or underlying conditions, while those without aspirin were generally healthier. These findings further raise the issue of whether the risk of hemorrhage in high-risk patients or patients with existing chronic diseases and continued aspirin therapy can be assumed equivalent to that in general patients who undergo similarly practiced oral surgery but do not take aspirin. A number of studies have investigated this issue and revealed conflicting results. Some studies have shown no significant difference in the risk of bleeding following dental extraction $[3,11,16]$, dental osteotomy [5], or minor oral surgical procedures [7] between patients who continue aspirin therapy and those who do not use aspirin. Others have contradictorily found an increased bleeding risk after dental extraction 
Table 4. Univariable and multivariable association of underlying systemic condition with continued aspirin therapy, other covariates, and bleeding

\begin{tabular}{|c|c|c|c|c|c|c|c|}
\hline \multirow{2}{*}{ Factors } & \multirow{2}{*}{$\begin{array}{c}\text { Bleeding } \\
\text { n }(\%)\end{array}$} & \multicolumn{3}{|c|}{ Univariable analysis } & \multicolumn{3}{|c|}{ Multivariable analysis } \\
\hline & & $\mathbf{R R}^{1}$ & $95 \% \mathrm{Cl}$ & $p$-value & $\mathbf{R R}$ & $95 \% \mathrm{Cl}$ & $p$-value \\
\hline Patients' group & & & & $<0.001$ & & & $<0.001$ \\
\hline Non-aspirin & $33(0.7)$ & 1.0 & Reference & & 1.0 & Reference & \\
\hline Aspirin & $37(5.8)$ & 7.7 & $4.8,12.4$ & & $4.5^{2}$ & $2.0,10.0$ & \\
\hline Sex & & & & 0.17 & & & 0.43 \\
\hline Male & $32(1.6)$ & 1.0 & Reference & & 1.0 & Reference & \\
\hline Female & $38(1.2)$ & 0.7 & $0.4,1.2$ & & 0.8 & $0.5,1.3$ & \\
\hline \multicolumn{8}{|l|}{ Age (y) } \\
\hline $20-59$ & $30(0.9)$ & 1.0 & Reference & & 1.0 & Reference & \\
\hline$\geq 60$ & $40(2.3)$ & 2.6 & $1.7,4.3$ & $<0.001$ & 1.1 & $0.6,1.9$ & 0.83 \\
\hline \multicolumn{8}{|l|}{ Diabetes } \\
\hline No & $46(1.0)$ & 1.0 & Reference & & 1.0 & Reference & \\
\hline Yes & $24(3.5)$ & 3.3 & $2.0,5.3$ & $<0.001$ & 0.8 & $0.4,1.6$ & 0.53 \\
\hline \multicolumn{8}{|l|}{ Hypertension } \\
\hline No & $32(0.8)$ & 1.0 & Reference & & 1.0 & Reference & \\
\hline Yes & $38(3.4)$ & 4.2 & $2.6,6.8$ & $<0.001$ & 1.5 & $0.8,3.0$ & 0.20 \\
\hline \multicolumn{8}{|l|}{ Cardiovascular disease } \\
\hline No & $57(1.1)$ & 1.0 & Reference & - & 1.0 & Reference & \\
\hline Yes & $13(8.8)$ & 7.3 & $4.0,13.4$ & $<0.001$ & 1.8 & $0.8,3.8$ & 0.14 \\
\hline \multicolumn{8}{|l|}{ Stroke } \\
\hline No & $67(1.3)$ & 1.0 & Reference & & 1.0 & Reference & \\
\hline Yes & $3(3.5)$ & 2.6 & $0.8,8.3$ & 0.11 & 0.8 & $0.2,2.7$ & 0.71 \\
\hline \multicolumn{8}{|l|}{ Asthma/COPD } \\
\hline No & $69(1.3)$ & 1.0 & Reference & & 1.0 & Reference & \\
\hline Yes & $1(1.2)$ & 0.9 & $0.1,6.8$ & 0.95 & 0.6 & $0.1,4.7$ & 0.67 \\
\hline \multicolumn{8}{|l|}{ Dental professional } \\
\hline Dental hygienist & $30(1.4)$ & 1.0 & Reference & & 1.0 & Reference & \\
\hline Dentist & $40(1.3)$ & 0.9 & $0.4,2.2$ & 0.81 & 1.0 & $0.5,2.1$ & 0.95 \\
\hline \multicolumn{8}{|l|}{ Surgical procedure } \\
\hline Simple extraction [+/- scaling or root planing] & $45(2.6)$ & 1.0 & Reference & & 1.0 & Reference & \\
\hline All other procedures & $25(0.7)$ & 0.3 & $0.2,0.4$ & $<0.001$ & 1.3 & $0.6,2.5$ & 0.51 \\
\hline \multicolumn{8}{|l|}{ No. of extracted teeth ${ }^{3}$} \\
\hline None & $4(0.2)$ & 1.0 & Reference & & & & \\
\hline 1 & $64(3.1)$ & 20.4 & $7.4,56.4$ & $<0.001$ & 1.0 & Reference & \\
\hline$\geq 2$ & $2(0.4)$ & 2.9 & $0.5,15.9$ & 0.22 & 0.1 & $0.0,0.5$ & 0.003 \\
\hline \multicolumn{8}{|l|}{ Only maxilla involved ${ }^{4}$} \\
\hline No & $48(1.3)$ & 1.0 & Reference & & & & \\
\hline Yes & $22(1.4)$ & 1.0 & $0.6,1.7$ & 0.90 & & & \\
\hline \multicolumn{8}{|l|}{ Only mandible involved } \\
\hline No & $22(0.6)$ & 1.0 & Reference & & 1.0 & Reference & \\
\hline Yes & $48(3.0)$ & 4.7 & $2.8,7.9$ & $<0.001$ & 2.3 & $1.3,3.9$ & 0.003 \\
\hline \multicolumn{8}{|l|}{ Position of involved teeth } \\
\hline Anterior segment only & $15(2.9)$ & 1.0 & Reference & & 1.0 & Reference & \\
\hline Premolar [+/- anterior] Segment & $9(1.7)$ & 0.6 & $0.3,1.3$ & 0.21 & 0.7 & $0.3,1.6$ & 0.35 \\
\hline Molar [+/- anterior or premolar] segment & $42(2.9)$ & 1.0 & $0.6,1.8$ & 1.00 & 1.3 & $0.7,2.6$ & 0.42 \\
\hline \multicolumn{8}{|l|}{ Visit } \\
\hline 1 (first) visit only & $43(1.5)$ & 1.0 & Reference & & 1.0 & Reference & \\
\hline$>1$ visit within period & $27(1.2)$ & 0.8 & $0.5,1.4$ & 0.49 & 0.9 & $0.5,1.4$ & 0.55 \\
\hline
\end{tabular}

$\mathrm{RR}$, risk ratio; $\mathrm{Cl}$, confidence interval; $\mathrm{COPD}$, chronic obstructive pulmonary disease.

${ }^{1}$ Crude RR estimated by multilevel Poisson regression.

${ }^{2}$ Adjusted RR estimated by multilevel Poisson regression adjusting for sex, age, diabetes, hypertension, cardiovascular disease, stroke, asthma/COPD, dental professional, surgical procedure, number of extracted teeth, procedures involving mandible, position of involved teeth, and visit.

${ }^{3}$ In multivariable model, the number of extracted teeth was categorized into two categories, 1 extracted tooth (reference category) and $\geq 2$ extracted teeth, to avoid collinearity problem. 'No extraction' category was adjusted in the model through 'Surgical procedure' variable.

${ }^{4}$ Variable was not included in multivariable model due to lack of association (crude $\mathrm{RR}=1.0, p=0.90$ ) and to avoid collinearity problem with 'Only mandible involved' variable. 
$[4,8,12]$ and minor surgical procedures [25], though the risk measures were not statistically significant $[8,12,25]$. In the present study, clinical evidence of an increased risk along with statistical significance (RR, $4.5 ; 95 \% \mathrm{Cl}, 2.0$ to $10.0 ; p<0.001)$ strongly suggested that bleeding following minor oral surgical procedures was more likely to occur in high-risk patients or patients with existing chronic diseases with continued aspirin therapy compared to general patients who did not use aspirin during surgery.

Since the study facility no longer practiced the discontinuation of aspirin use before minor oral surgery, a comparison group consisting of patients with similar risks or existing chronic diseases and discontinued aspirin therapy before oral surgery could not be acquired. Therefore, this study instead compared the bleeding risk following oral surgery in patients on continuous aspirin therapy with that in a group not on aspirin at all [8]. Patients in the non-aspirin group were evidently healthier than those in the aspirin group as indicated by considerably smaller proportions of patients in the non-aspirin group with advanced age, diabetes, hypertension, cardiovascular disease, or stroke (Table 1). Nonetheless, the marked difference in patients' baseline clinical characteristics should not be considered a consequence of control selection bias since patients in both groups underwent similarly practiced minor oral surgery with an assumed equivalent risk of bleeding $[3,5,7,11,16]$. This design, therefore, allowed for the evaluation of this clinical assumption and upheld the safety of current recommendations [1-16].

Analysis of the bleeding occurrence in patients who made either single or multiple visits and underwent a variety of oral surgical procedures in a practical setting could provide realworld estimates of the overall bleeding risk, in lieu of a procedure-specific risk. This could be generalized to the source population to which these patients represent and could be useful for risk communication to patients who continue aspirin therapy before oral surgery in general. In addition, a comprehensive assessment of risk characteristics for bleeding should always be undertaken before oral surgery on a case-by-case basis, since bleeding can be influenced by a combination of several factors [1], such as a patient's inherent characteristics, chronic disease, medication, procedural invasiveness, and biological variations in bleeding potential. In this study, the increased bleeding risk in the aspirin group should not be considered as an effect of continued aspirin therapy alone. In spite of the effort to control the effects of known and ascertainable chronic diseases through a regression modeling approach, unmeasured systemic conditions, such as the severity and duration of chronic diseases or obesity [1], could be an alternative explanation for the increased risk. Therefore, the increased bleeding risk observed in this study could be a combined effect of high-risk conditions or existing chronic diseases and continued aspirin therapy. With respect to the differing minor oral surgical procedures, a previous study suggested that bleeding risk was independent of the type of procedure performed [7]. In contrast, this study determined that there was a significant difference in the bleeding risk between the two groups when stratified by simple versus complicated extractions (Table 3). Following the mildly invasive scaling procedure, bleeding was observed in both groups in this study. This contradicted a previous finding that scaling did not contribute to bleeding [25]. Additionally, the difference in bleeding risk for patients who undergo surgical removal of impacted teeth could not be assessed since none of the patients in the aspirin group underwent this invasive procedure. With respect to the interaction effect of aspirin use with each of the other prognostic factors on the bleeding outcome, these interactions were explored by statistical means in addition to the stratified analysis. Bleeding risk was uniformly observed across stratified subgroups of each prognostic factor. However, one exception was found among patients who underwent minor surgery involving the mandible only, where the risk of bleeding was significantly higher in the aspirin group. This preliminary result suggested the need for further study devoted to determining the interaction effects of aspirin therapy with these prognostic factors (particularly minor surgery involving the mandible only) on bleeding from both a statistical and clinical perspective in order to provide a rational clinical explanation.

All bleeding events were effectively controlled by local hemostatic measures comprising a pressure pack and suturing either with or without additional use of a sterile compressed sponge (Gelfoam). Life-threatening bleeding complications were not found in this or other studies [3-16,25]. Since oral bleeding can be visible and promptly managed [5], minor oral surgical procedures can be cautiously provided without the discontinuation of aspirin therapy, thereby minimizing the risk of thromboembolism [1]. Preventive strategies, including preoperative risk communication and perception, reductions in tissue damage during surgery, prepared hemostatic measures, and postoperative monitoring for bleeding would ensure that minor oral surgery is safe for high-risk patients and patients 
with existing chronic diseases who continue aspirin use.

In conclusion, high-risk patients and patients with existing chronic diseases who continued aspirin use were at a higher risk of hemorrhage following minor oral surgery compared to those who were generally healthier and did not use aspirin. Nonetheless, the bleeding complications were not life-threatening and could be promptly managed through simple hemostatic measures. Minor oral surgery can therefore be provided as long as it is accompanied by an awareness of the increased bleeding risk, prepared hemostatic measures, and postoperative monitoring of bleeding. This negates the need to discontinue aspirin therapy, which may lead to more serious complications.

\section{ACKNOWLEDGEMENTS}

The authors would like to acknowledge Huayploo Hospital, which provided official permission to use its routine clinical data for this investigation. Appreciation is also extended to Ms. Jalisa Gilmore, MPH, for her proofreading and comments during manuscript preparation.

\section{CONFLICT OF INTEREST}

The authors have no conflicts of interest associated with the material presented in this paper.

\section{ORCID}

Chanapong Rojanaworarit http://orcid.org/0000-0001-70097416

Soontaree Limsawan http://orcid.org/0000-0002-0587-8512

\section{REFERENCES}

1. Verma G. Dental extraction can be performed safely in patients on aspirin therapy: a timely reminder. ISRN Dent 2014; 2014:463684.

2. Ringel R, Maas R. Dental procedures in patients treated with antiplatelet or oral anticoagulation therapy: an anonymous survey. Gerodontology 2016;33(4):447-452.

3. Bajkin BV, Urosevic IM, Stankov KM, Petrovic BB, Bajkin IA. Dental extractions and risk of bleeding in patients taking single and dual antiplatelet treatment. Br J Oral Maxillofac Surg 2015;53(1):39-43.

4. Zhao B, Wang P, Dong Y, Zhu Y, Zhao H. Should aspirin be stopped before tooth extraction? A meta-analysis. Oral Surg Oral Med Oral Pathol Oral Radiol 2015;119(5):522-530.

5. Hanken H, Tieck F, Kluwe L, Smeets R, Heiland M, Precht C, et al. Lack of evidence for increased postoperative bleeding risk for dental osteotomy with continued aspirin therapy. Oral Surg Oral Med Oral Pathol Oral Radiol 2015;119(1):17-19.

6. Wahl MJ. Dental surgery and antiplatelet agents: bleed or die. Am J Med 2014;127(4):260-267.

7. Girotra C, Padhye M, Mandlik G, Dabir A, Gite M, Dhonnar R, et al. Assessment of the risk of haemorrhage and its control following minor oral surgical procedures in patients on antiplatelet therapy: a prospective study. Int J Oral Maxillofac Surg 2014;43(1):99-106.

8. Lillis T, Ziakas A, Koskinas K, Tsirlis A, Giannoglou G. Safety of dental extractions during uninterrupted single or dual antiplatelet treatment. Am J Cardiol 2011;108(7):964-967.

9. Napeñas JJ, Oost FC, DeGroot A, Loven B, Hong CH, Brennan MT, et al. Review of postoperative bleeding risk in dental patients on antiplatelet therapy. Oral Surg Oral Med Oral Pathol Oral Radiol 2013;115(4):491-499.

10. Verma G, Tiwari AK, Chopra S. Aspirin and exodontia: a comparative study of bleeding complications with aspirin therapy. Int J Dent Sci Res 2013;1(2):50-53.

11. Sadeghi-Ghahrody M, Yousefi-Malekshah SH, Karimi-Sari H, Yazdanpanah H, Rezaee-Zavareh MS, Yavarahmadi M. Bleeding after tooth extraction in patients taking aspirin and clopidogrel (Plavix ${ }^{\oplus}$ ) compared with healthy controls. Br J Oral Maxillofac Surg 2016;54(5):568-572.

12. Lu SY, Tsai CY, Lin LH, Lu SN. Dental extraction without stopping single or dual antiplatelet therapy: results of a retrospective cohort study. Int J Oral Maxillofac Surg 2016;45(10):12931298.

13. Schreuder WH, Peacock ZS. Antiplatelet therapy and exodontia. J Am Dent Assoc 2015;146(11):851-856.

14. Napeñas JJ, Hong CH, Brennan MT, Furney SL, Fox PC, Lockhart PB. The frequency of bleeding complications after invasive dental treatment in patients receiving single and dual antiplatelet therapy. J Am Dent Assoc 2009;140(6):690-695.

15. van Diermen DE, van der Waal I, Hoogstraten J. Management recommendations for invasive dental treatment in patients using oral antithrombotic medication, including novel oral anticoagulants. Oral Surg Oral Med Oral Pathol Oral Radiol 2013;116(6):709-716.

16. Nooh N. The effect of aspirin on bleeding after extraction of teeth. Saudi Dent J 2009;21(2):57-61. 
17. Pignone M, Alberts MJ, Colwell JA, Cushman M, Inzucchi SE, Mukherjee $D$, et al. Aspirin for primary prevention of cardiovascular events in people with diabetes: a position statement of the American Diabetes Association, a scientific statement of the American Heart Association, and an expert consensus document of the American College of Cardiology Foundation. Circulation 2010;121(24):2694-2701.

18. Krishnan B, Shenoy NA, Alexander M. Exodontia and antiplatelet therapy. J Oral Maxillofac Surg 2008;66(10):20632066.

19. Wears RL. Advanced statistics: statistical methods for analyzing cluster and cluster-randomized data. Acad Emerg Med 2002;9(4):330-341.

20. Zou G. A modified poisson regression approach to prospective studies with binary data. Am J Epidemiol 2004;159(7): 702-706.

21. Karimi-Sari H, Rezaee-Zavareh MS. Pharmacology: confound- ers for bleeding. Br Dent J 2016;220(12):611.

22. Nansseu JR, Noubiap JJ. Aspirin for primary prevention of cardiovascular disease. Thromb J 2015;13:38.

23. Collet JP, Montalescot G, Blanchet B, Tanguy ML, Golmard JL, Choussat $\mathrm{R}$, et al. Impact of prior use or recent withdrawal of oral antiplatelet agents on acute coronary syndromes. Circulation 2004;110(16):2361-2367.

24. Antithrombotic Trialists' (ATT) Collaboration, Baigent C, Blackwell L, Collins R, Emberson J, Godwin J, et al. Aspirin in the primary and secondary prevention of vascular disease: collaborative meta-analysis of individual participant data from randomised trials. Lancet 2009;373(9678):1849-1860.

25. Tientaworn I, Rojanaworarit C. Risk of uncontrolled bleeding after oral surgical procedures attributed to chronic conditions and antiplatelet therapy: a practice-based analysis of patients attending a district hospital in Thailand. Reg 4-5 Med J 2016; 35(1):36-44. 